\title{
Periprosthetic Joint Infection Does Not Preclude Good Outcomes after a Revision Total Knee Arthroplasty: A 7-Year Follow-Up Study of 144 Retrospective Cases
}

\author{
Du Hyun Ro, Jong-Keun Kim, Sunghwan Kim, Hyuk-Soo Han, and Myung Chul Lee \\ Department of Orthopaedic Surgery, Seoul National University College of Medicine, Seoul, Republic of Korea \\ Correspondence should be addressed to Myung Chul Lee; leemc@snu.ac.kr
}

Received 7 November 2017; Revised 8 May 2018; Accepted 11 July 2018; Published 12 August 2018

Academic Editor: Bernd Fink

Copyright (C) 2018 Du Hyun Ro et al. This is an open access article distributed under the Creative Commons Attribution License, which permits unrestricted use, distribution, and reproduction in any medium, provided the original work is properly cited.

\begin{abstract}
Background and Purpose. Debate exists on whether septic revision total knee arthroplasty (TKA) results in inferior clinical outcomes, and limited information is available regarding the factors associated with such outcomes. This study aimed to (1) compare clinical outcomes and characteristics of aseptic versus septic revision TKA and (2) identify the risk factors associated with inferior clinical outcomes. Methods. We retrospectively reviewed 144 revision TKAs (90 aseptic and 54 septic revisions) that were followed for a minimum of 3 years (mean $=7$ years). Clinical outcome data, namely, Knee Society knee and function scores and the Hospital for Special Surgery knee score, were collected. We reviewed 13 pre- and intraoperative variables. Results. Postoperative clinical outcomes were inferior in septic revision surgeries $(p<0.05)$. In regression analyses, however, septic revision was not an independent risk factor for poor clinical outcomes. The independent risk factors for poor outcome were identified where Anderson Orthopedic Research Institute grade 3 femoral and tibial bone defects, more than three surgeries, and treatment for persistent infection were associated with inferior clinical outcomes (all $p<0.05$ ). Standard two-stage septic revision without grade 3 bone defects or additional surgeries showed comparable outcomes to aseptic revision. Interpretation. Clinical outcomes of septic revision were inferior to those of aseptic revision. However, poor outcomes were mainly associated with large bone defects and an increased number of surgeries. The outcomes of aseptic and septic revision surgery were similar when patients with larger bone defects and more than three surgeries were excluded.
\end{abstract}

\section{Introduction}

More than 650,000 total knee arthroplasties (TKAs) are performed annually in the United States [1]. As the geriatric population increases, the number of TKAs is expected to increase; subsequently, the demand for revision surgery will also increase [1]. Revision surgery is a complex, demanding procedure and, importantly, clinical outcomes are less satisfying than those of primary TKA $[2,3]$.

It is generally accepted that the etiology of revision surgery influences the outcome. Of the major etiologies for revision surgery, septic revision is associated with the worst outcome [2-8]. Before the introduction of two-stage revision surgeries, eradication of infection was less common; and consequently clinical outcomes were rather poor [9].

As treatment strategies for septic revision have improved, identification of organisms, eradication rates, and clinical outcomes have also improved impressively [10-14]. Some authors have reported that septic and aseptic revision groups have had similar outcomes regarding pain, functional scores, survival, and mental health status [10-12]. Patil et al. even reported a higher clinical score with septic versus aseptic revisions [11]. Recent literature has suggested that when a standard protocol and team-based approach are used, periprosthetic infection does not preclude a good outcome after revision TKA [10-12]. Hence, there is now debate regarding whether septic revision is associated with poor clinical outcomes.

Such debate regarding the clinical outcomes of septic versus aseptic surgery suggested to us that focusing on the "cause of revision" may mean that something more important is missed. Of note, revision surgeries comprise diverse clinical situations. Unlike primary TKA, revision surgeries are associated with various degrees of bone defect, different 


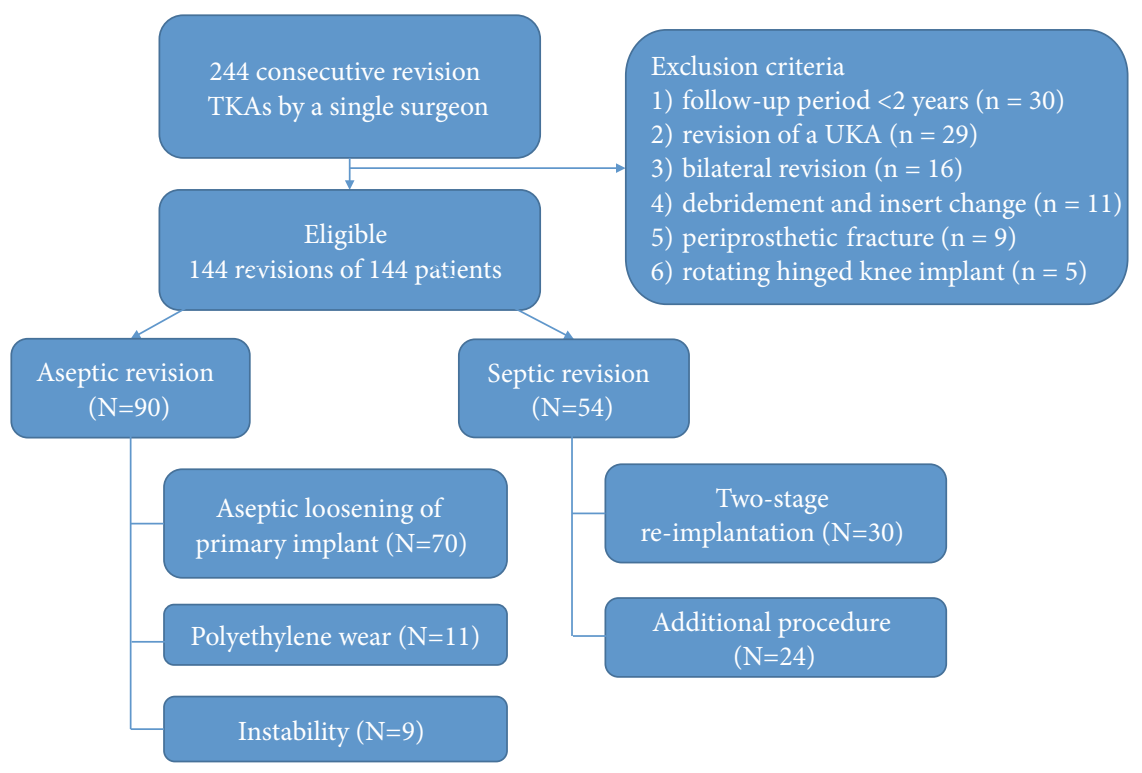

Figure 1: Flow chart of the study subjects.

implant configurations, the use of a more extensive surgical approach, and the need for repeat surgeries [7, 15, 16]. Hence, we hypothesized that the cause of the revision, especially infection, could be a confounding factor and that other unknown factors associated with infection could be more directly related to clinical outcomes. However, limited information is currently available regarding the factors associated with inferior clinical outcomes in revision TKAs with midto long-term follow-up. Information regarding this subject would help to improve the clinical outcome of revision TKA. Therefore, this study aimed to (1) compare clinical outcomes of aseptic versus septic revision TKA and (2) identify risk factors associated with inferior clinical outcomes.

\section{Materials and Methods}

2.1. Study Subjects. This retrospective study was approved by our local Institutional Review Board (Protocol No: 1307114-506). We reviewed a single institution database of 244 consecutive revision TKAs performed by a single surgeon from 1995 to 2015. Based on the following criteria, 100 revisions were excluded: (1) follow-up period less than 2 years or loss $(n=30)$; (2) revision of a unicompartmental knee arthroplasty $(n=29)$; (3) bilateral revision $(n=16)$; (4) acute hematogenous infection that was successfully treated with debridement and insert change $(n=11)$; (5) periprosthetic fracture that required revision TKA $(n=9)$; and $(6)$ rotating hinged knee implant $(n=5)$. This left 144 revisions of 144 patients (Figure 1). The study group included 20 males and 124 females with an average age of 68.4 years (range: $50-83 \pm 7.2$ years). The aseptic revision group included 70 cases with aseptic loosening of the primary implant, 11 cases with polyethylene wear, and 9 cases with instability. Both component revisions (Femur and Tibial component) were performed for all aseptic revisions. The septic revision group included 30 cases with chronic infection that were treated with two-stage reimplantation and 24 cases that underwent an additional arthrotomy and debridement for persistent infection, before or after two-stage reimplantation.

The average length of follow-up after revision was $84 \pm$ 28.7 months (range: 40-168 months), and the average interval between the primary and revision surgeries was $99 \pm 58.7$ months.

During the study period, revision surgery was performed with either a varus-valgus constrained implant (LCCK ${ }^{\circledR}$, NexGen ${ }^{\circledR}$, Zimmer, Warsaw, IN, USA) or a posterior stabilized implant (LPS ${ }^{\circledR}, \mathrm{NexGen}^{\circledR}$, Zimmer, Warsaw, IN, USA), depending on the stability [16]. A fluted titanium extension stem, titanium block, and/or strut allograft were used, depending on the bone defect. Contained bone defects $<5 \mathrm{~mm}$ thick were filled with bone cement. Uncontained bone defects $\leq 10 \mathrm{~mm}$ thick were treated with block augment and uncontained bone defects $>10 \mathrm{~mm}$ thick were treated with strut allografts using screw fixation (Figure 2). Stem extensions were fixed using the hybrid fixation technique for the entire implant. Intraoperative observations were systemically collected using a predesigned database. Bone defects were classified according to the Anderson Orthopedic Research Institute (AORI) bone defect protocol [17]. Two independent investigators prospectively collected all the clinical information using the predesigned computer database (SMA and EMS). Basic demographic data and clinical outcomes, including the Knee Society Knee score (KSKS) and Knee Society function score (KSFS), and the Hospital for Special Surgery knee score (HSS) were recorded. Postrevision outcomes were collected annually and the most recent follow-up data were used. ROM was measured from maximum extension to maximum flexion using a standard clinical goniometer with the patient in the supine position.

2.2. Protocol of Septic Revision Surgery. For patients with a chronic periprosthetic infection, a two-stage reimplantation 


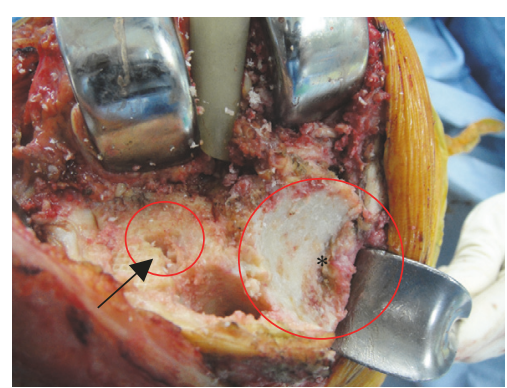

(a)

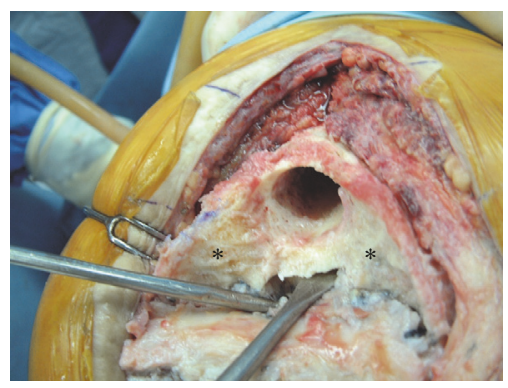

(d)

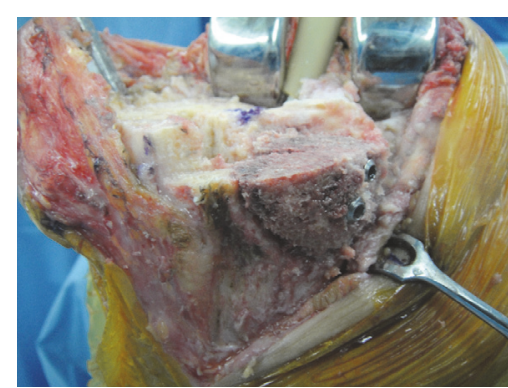

(b)

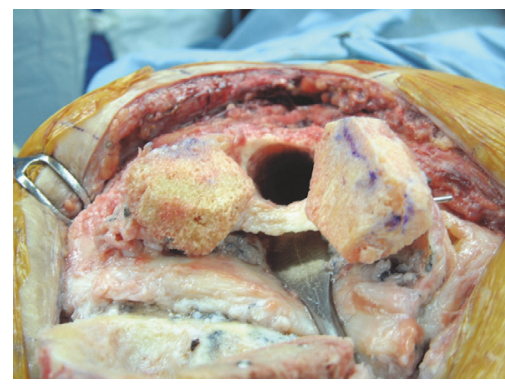

(e)

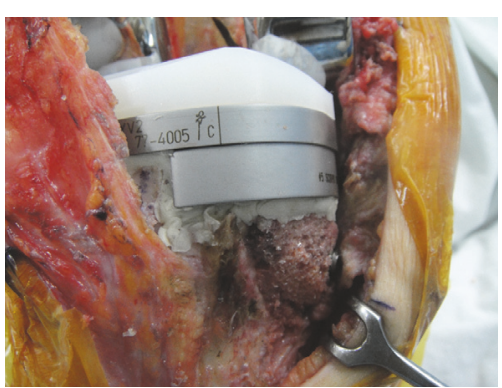

(c)

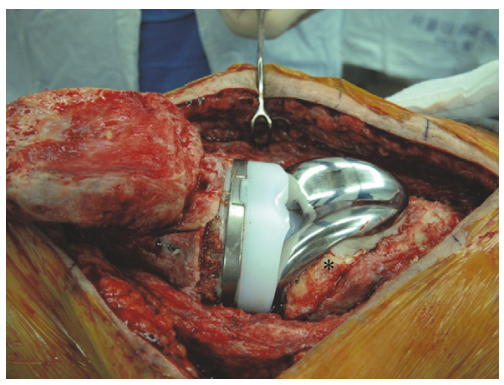

(f)

FIGURE 2: Bone defect filling process. (a) Intraoperative photograph showing an unconstrained, large bone defect with destruction of the metaphyseal bone (grade 3 bone defect) on the medial side of the tibia (asterisk) and a small, constrained bone defect (arrow) on the lateral side of the tibia. (b) Initially the large bone defect was treated with allografts using screw fixation in the metaphysis. (c) Then the remaining unconstrained bone defect was filled with a metal block and the defect on the lateral side was filled with cement. (d) Intraoperative photograph showing unconstrained large bone defect (asterisks) with destruction of major metaphyseal bone in femur (grade 3 bone defect). (e) Allograft was also used to fill the large bone defect. (f) Implant was inserted to the augmented area and the allograft was tightly compacted between femur component and remaining bone (asterisk).

was performed, which included removal of components, extensive debridement, and placement of an antibioticimpregnated articulating cement spacer, followed by 6 to 8 weeks of intravenous antibiotics according to the microorganism. After a 4-week antibiotic free interval, we performed laboratory tests and joint aspirations to determine whether the infection has been eradicated. If so, reimplantation was performed. Debridement and replacement of the antibioticimpregnated cement spacer were performed instead of reimplantation if there were signs and symptoms of persistent infection. The criteria for a persistent infection included ongoing discharge and erythema, higher than $36.5^{\circ} \mathrm{C}$ of the body temperature, higher than $0.5 \mathrm{mg} / \mathrm{dl}$ of the Creactive protein level, or more than five polymorphonuclear neutrophils observed on any high-power field (HPF) in 10 frozen section specimens harvested intraoperatively from the synovium or necrotic tissue debris.

2.3. Statistical Analysis. Clinical outcomes and characteristics of the septic revisions were compared with those of the aseptic revisions using Student's t-test for continuous, normally distributed data and Pearson's chi-square test for nominal, categorical data. Within both groups, the prerevision and postrevision data were compared using the paired $t$ test for the normally distributed data. Normality of data was assessed using the Kolmogorov-Smirnov test. For all analyses, the level of significance was set at a $p$ value of $<0.05$.
To identify the factors associated with inferior clinical outcomes in revision TKAs, linear regression analyses were used. Thirteen variables were assessed, including age, sex, body mass index (BMI), the primary diagnosis (0, osteoarthritis; 1 , rheumatoid arthritis), the cause (0, aseptic loosening; 1, septic loosening), prerevision ROM, implant type (0, PS implant; 1, LCCK), the surgical approach $(0$, standard parapatellar approach; 1, quad-snip; 2, V-Y quadriceps plasty; and 3, tibial tubercle osteotomy), the femur bone defect (AORI type 1,2, or 3 ), the tibia bone defect (AORI type 1,2 , or 3 ), complications ( 0 , no complication; 1 , a complication present), insert thickness, and number of operations (arthrotomy operation). Factors with a $\mathrm{p}$ value $<0.20$ on univariate analysis were assessed subsequently through multivariate analysis using the stepwise method. Statistical analyses were performed using the SPSS ${ }^{\circledR}$ for Windows ${ }^{\circledR}$ statistical software package (ver. 19.0.1; SPSS Inc., Chicago, IL, USA).

\section{Results}

The preoperative clinical outcomes were similar between the aseptic versus septic revision groups (Figure 3). However, the preoperative $\mathrm{ROM}$ was higher in the aseptic revision group $(\mathrm{p}<0.001)$. In both groups, all clinical outcomes (KSKS, KSFS, and HSS scores) improved after revision $(p<0.001)$ as did the ROM. However, the final scores were less satisfying in the 
TABLE 1: Characteristics of the study groups.

\begin{tabular}{|c|c|c|c|}
\hline & $\begin{array}{l}\text { Aseptic revision } \\
\quad(\mathrm{n}=90) \\
\text { Mean } \pm \mathrm{SD}\end{array}$ & $\begin{array}{l}\text { Septic revision } \\
\qquad(\mathrm{n}=54) \\
\text { Mean } \pm \text { SD }\end{array}$ & P-value \\
\hline Age (years) & $69.1(50-83)$ & $67.2(50-80)$ & 0.095 \\
\hline Female gender & $85(94.4 \%)$ & $39(72.2 \%)$ & $<0.001$ \\
\hline Body mass index $\left(\mathrm{kg} / \mathrm{m}^{2}\right)$ & $28.0 \pm 4.4$ & $25.9 \pm 3.6$ & 0.007 \\
\hline Average polyethylene thickness (mm) & $16.6 \pm 3.29$ & $16.2 \pm 3.3$ & 0.621 \\
\hline Varus-valgus constrained implant & $61(67.8 \%)$ & $50(92.6 \%)$ & $<0.001$ \\
\hline \multicolumn{4}{|l|}{ Surgical approach } \\
\hline Standard parapatellar approach & $64(71.1 \%)$ & $26(48.1 \%)$ & \multirow{2}{*}{0.008} \\
\hline Extensive approach & $26(28.9 \%)$ & $28(51.9 \%)$ & \\
\hline \multicolumn{4}{|l|}{ Bone defect } \\
\hline Grade 1 / 2 / 3 femoral bone defect & $30 / 55 / 5$ & $4 / 37 / 13$ & $<0.001$ \\
\hline Grade 1 / 2 / 3 tibial bone defect & $32 / 53 / 5$ & $20 / 27 / 7$ & 0.256 \\
\hline Average number of surgeries & 1.01 & $2.7 *$ & $<0.001$ \\
\hline Wound complications & 1 case & 6 cases & 0.011 \\
\hline Average time interval between primary and revision surgery (months) & $127 \pm 35$ & $53 \pm 28$ & $<0.001$ \\
\hline
\end{tabular}

Values are means \pm standard deviations or percentages.

* Two-stage revision, 30 cases; three- or four-stage revision, 24 cases.

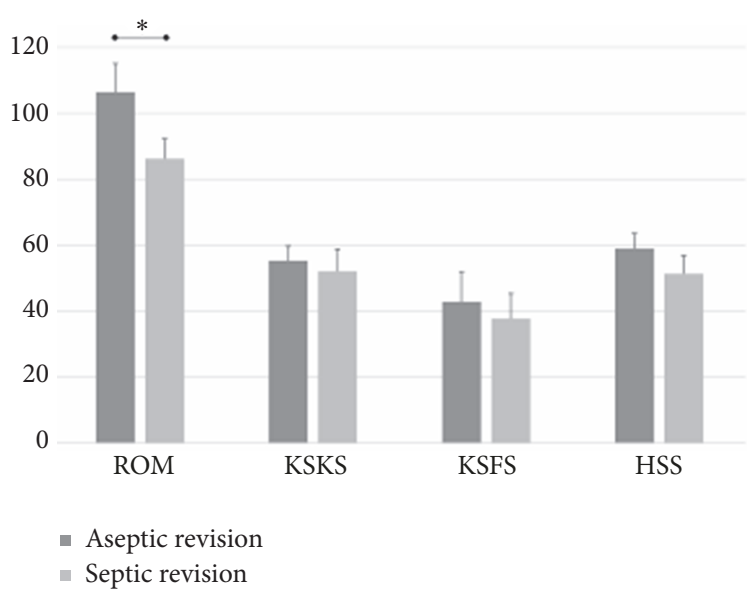

FIGURE 3: Characteristics and clinical scores of each revision group. Preoperative ROM was significantly greater in the aseptic revision group. Values are means and standard deviations, and the asterisk denotes statistical significance. ROM, range of motion; KSKS, Knee Society knee score; HSS, Hospital for Special Surgery knee score.

septic revision group. The postrevision ROM, KSKS score, and HSS score were significantly lower $(p=0.030,<0.001$, and 0.003 , respectively). Only the KSFS score was similar between the two groups ( $\mathrm{p}=0.105$, Figure 4 ).

Regarding the pre- and intraoperative factors, constrained implant was more frequently used in the septic revision group $(\mathrm{p}<0.001)$ and a more extensive approach was chosen $(p=0.008)$ (Table 1$)$. Repeated surgery was required in the septic revision group $(p<0.001)$ and complications were more frequent $(\mathrm{p}=0.011)$. Also, the femoral bone defects tended to be more extensive $(p<0.001)$.

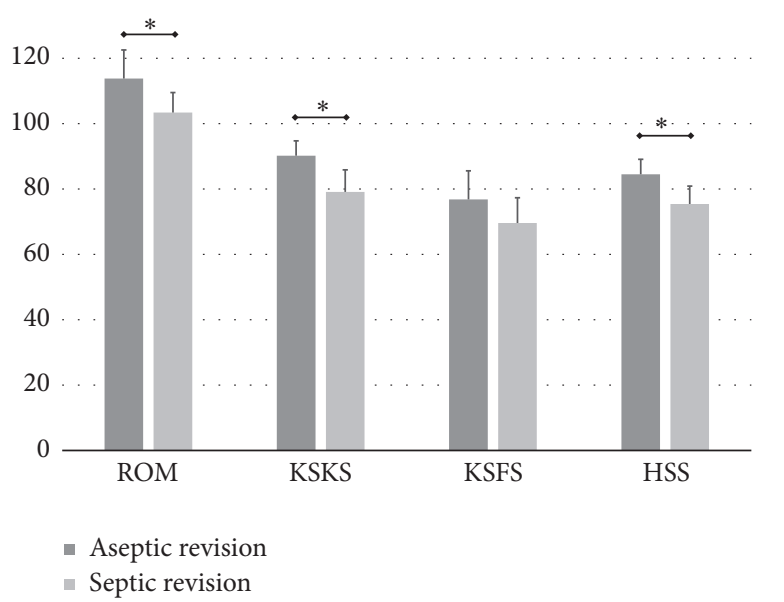

FIgUre 4: Postoperative outcomes of each revision group. Postoperative ROM, KSKS, and HSS were significantly lower in the septic revision group. Values are means and standard deviations, and asterisks denote statistical significance. ROM, range of motion; KSKS, Knee Society knee score; HSS, Hospital for Special Surgery knee score.

Univariate and subsequent multivariate linear regression analyses were performed to identify factors that were associated with clinical outcomes and ROM (Tables 2 and 3). Regression analysis revealed that the postrevision ROM increased with age $(p=0.001)$ and greater prerevision ROM $(p<0.001)$ and decreased with tibia bone defects (both grades 2 and $3, p<0.001$ and $p<0.002$, respectively) and three or four surgeries $(p<0.001)$. We performed the same analysis for the remaining clinical outcomes and showed that femoral bone defects (grade 3), tibial bone defects (grade 3 ), and the three or four surgeries were strongly associated with inferior 
TABLE 2: Results of univariate regression analysis including 13 pre- and intraoperative variables and four clinical outcomes.

\begin{tabular}{|c|c|c|c|c|c|c|c|c|}
\hline \multirow[b]{2}{*}{ Variable } & \multicolumn{2}{|c|}{ ROM } & \multicolumn{2}{|c|}{ KSKS } & \multicolumn{2}{|c|}{ KSFS } & \multicolumn{2}{|c|}{ HSS } \\
\hline & $\beta \pm \mathrm{SE} *$ & P-value & $\beta \pm \mathrm{SE} *$ & $\mathrm{P}$-value & $\beta \pm \mathrm{SE} *$ & P-value & $\beta \pm \mathrm{SE} *$ & $\mathrm{P}$-value \\
\hline (1) Age & $0.8 \pm 0.2$ & $<0.001$ & $0.1 \pm 0.2$ & 0.339 & $0 \pm 0.2$ & 0.891 & $0.2 \pm 0.2$ & 0.233 \\
\hline (2) Female gender & $1.5 \pm 4.6$ & 0.742 & $3.9 \pm 3.2$ & 0.227 & $0.6 \pm 4.3$ & 0.885 & $0.7 \pm 3.3$ & 0.842 \\
\hline (3) Body mass index & $0.5 \pm 0.4$ & 0.259 & $0 \pm 0.3$ & 0.996 & $0 \pm 0.4$ & 0.919 & $-0.1 \pm 0.3$ & 0.837 \\
\hline \multicolumn{9}{|l|}{$\begin{array}{l}\text { (4) Primary diagnosis } \\
\text { Osteoarthritis (comparator) }\end{array}$} \\
\hline Rheumatoid arthritis & $-7.2 \pm 6.6$ & 0.279 & $-1.9 \pm 4.8$ & 0.697 & $-7.5 \pm 6.5$ & 0.249 & $-7.4 \pm 4.9$ & 0.132 \\
\hline Other & $-3.6 \pm 8$ & 0.659 & $3 \pm 5.5$ & 0.590 & $2.2 \pm 7.5$ & 0.771 & $4.5 \pm 5.6$ & 0.425 \\
\hline (5) Cause & $-5.3 \pm 1.6$ & 0.001 & $-4.8 \pm 1.1$ & $<0.001$ & $-3.7 \pm 1.5$ & 0.017 & $-4.7 \pm 1.1$ & $<0.001$ \\
\hline (6) Preoperative ROM & $0.3 \pm 0.1$ & $<0.001$ & $0.1 \pm 0$ & 0.014 & $0.1 \pm 0.1$ & 0.028 & $0.1 \pm 0$ & 0.016 \\
\hline (7) Implant & $-2.8 \pm 3.8$ & 0.460 & $-3.8 \pm 2.6$ & 0.145 & $-8.4 \pm 3.5$ & 0.016 & $-5.6 \pm 2.6$ & 0.034 \\
\hline \multicolumn{9}{|c|}{ (8) Standard paramedian approach (comparator) } \\
\hline Quadriceps snip & $-7 \pm 3.6$ & 0.056 & $-6.9 \pm 2.5$ & 0.006 & $-10.5 \pm 3.3$ & 0.002 & $-5.2 \pm 2.6$ & 0.046 \\
\hline VY quadriceps plasty & $-1 \pm 5.8$ & 0.866 & $7.3 \pm 3.9$ & 0.066 & $11.2 \pm 5.3$ & 0.037 & $8 \pm 4$ & 0.049 \\
\hline Tibial tubercle osteotomy & $-8.4 \pm 6.6$ & 0.207 & $-5.7 \pm 4.5$ & 0.208 & $1.2 \pm 6.2$ & 0.85 & $-7.7 \pm 4.9$ & 0.118 \\
\hline \multicolumn{9}{|c|}{ (9) Grade 1 femur bone defect (comparator) } \\
\hline Grade 2 & $-5.1 \pm 3.3$ & 0.125 & $0.9 \pm 2.3$ & 0.696 & $-2.6 \pm 3.1$ & 0.397 & $0.1 \pm 2.3$ & 0.955 \\
\hline Grade 3 & $-21.5 \pm 4.8$ & $<0.001$ & $-21.6 \pm 3.1$ & $<0.001$ & $-18.5 \pm 4.5$ & $<0.001$ & $-18.7 \pm 3.2$ & $<0.001$ \\
\hline \multicolumn{9}{|c|}{ (10) Grade 1 tibial bone defect (comparator) } \\
\hline Grade 2 & $-10 \pm 3.1$ & 0.002 & $-4.3 \pm 2.2$ & 0.051 & $-8.6 \pm 2.9$ & 0.018 & $-5.2 \pm 2.2$ & 0.022 \\
\hline Grade 3 & $-20.8 \pm 6.4$ & 0.001 & $-13.7 \pm 4.4$ & 0.002 & $-13.7 \pm 6.1$ & 0.026 & $-11.8 \pm 4.8$ & 0.016 \\
\hline (11) Insert thickness & $0.4 \pm 0.6$ & 0.499 & $0.1 \pm 0.4$ & 0.780 & $0.3 \pm 0.5$ & 0.562 & $0 \pm 0.4$ & 0.978 \\
\hline (12) Wound complications & $-16.6 \pm 7.9$ & 0.038 & $-8.7 \pm 5.5$ & 0.115 & $-4.4 \pm 7.5$ & 0.555 & $-18.8 \pm 5.4$ & 0.001 \\
\hline \multicolumn{9}{|c|}{ (13) Single surgery (comparator) } \\
\hline Two-stage & $5.2 \pm 3.9$ & 0.19 & $-2.1 \pm 2.7$ & 0.448 & $-5.2 \pm 3.7$ & 0.164 & $1.5 \pm 2.8$ & 0.584 \\
\hline Three- or four-stage & $-22.3 \pm 3.8$ & $<0.001$ & $-15.5 \pm 2.7$ & $<0.001$ & $-7.9 \pm 3.9$ & 0.025 & $-16.9 \pm 2.6$ & $<0.001$ \\
\hline
\end{tabular}

* Values are standardized regression coefficients $(\beta) \pm$ standard errors (SE). ROM, range of motion; KSKS, Knee Society knee score; HSS, Hospital for Special Surgery knee score; KSFS, Knee Society function score

TABLE 3: Results of multivariate regression analysis: relationships between selected variables and four clinical outcomes*.

\begin{tabular}{|c|c|c|c|c|c|c|c|c|}
\hline \multirow{2}{*}{$\begin{array}{l}\text { Multivariate analysis } \\
\text { Variable }\end{array}$} & \multicolumn{2}{|c|}{ ROM } & \multicolumn{2}{|c|}{ KSKS } & \multicolumn{2}{|c|}{ KSFS } & \multicolumn{2}{|c|}{ HSS } \\
\hline & $\beta \pm \mathrm{SE}^{\dagger}$ & $\mathrm{P}$-value & $\beta \pm \mathrm{SE}^{\dagger}$ & $\mathrm{P}$-value & $\beta \pm \mathrm{SE}^{\dagger}$ & $\mathrm{P}$-value & $\beta \pm \mathrm{SE}^{\dagger}$ & P-value \\
\hline Age & $0.6 \pm 0.2$ & 0.001 & & & & & & \\
\hline \multicolumn{9}{|l|}{ Cause of revision } \\
\hline Preoperative ROM & $0.2 \pm 0.1$ & $<0.001$ & & & & & & \\
\hline \multicolumn{9}{|c|}{ Grade 1 femur bone defect (comparator) } \\
\hline Grade 2 & & & $-5.8 \pm 2.0$ & 0.005 & $-10.2 \pm 3.2$ & 0.002 & & \\
\hline Grade 3 & & & $-21.6 \pm 3.4$ & $<0.001$ & $-25.6 \pm 4.9$ & $<0.001$ & $-12.9 \pm 3.1$ & $<0.001$ \\
\hline \multicolumn{9}{|c|}{ Grade 1 tibial bone defect (comparator) } \\
\hline Grade 2 & $-12.9 \pm 2.5$ & $<0.001$ & & & & & & \\
\hline Grade 3 & $-16.7 \pm 5.3$ & 0.002 & & & & & $-5.5 \pm 1.9$ & 0.004 \\
\hline \multicolumn{9}{|c|}{ Single surgery (comparator) } \\
\hline \multicolumn{9}{|l|}{ Two-stage } \\
\hline Three- or four-stage & $-16.1 \pm 3.3$ & $<0.001$ & $-10.2 \pm 2.5$ & $<0.001$ & & & $-13.3 \pm 2.5$ & $<0.001$ \\
\hline $\mathrm{R}_{\text {adj }}^{2}$ & \multicolumn{2}{|c|}{0.45} & \multicolumn{2}{|c|}{0.36} & \multicolumn{2}{|c|}{0.16} & \multicolumn{2}{|c|}{0.34} \\
\hline
\end{tabular}

$*$ Variables with $\mathrm{p}<0.20$ in the univariate analysis were included in the multivariate analysis (stepwise method). Nonsignificant factors were excluded from the table. ROM, range of motion; KSKS, Knee Society knee score; HSS, Hospital for Special Surgery knee score.

${ }^{\dagger}$ Values are $\beta \pm \mathrm{SE}$.

${ }^{\ddagger} \mathrm{R}^{2}{ }_{\text {adj }}$, percent variance explained by each variable. 


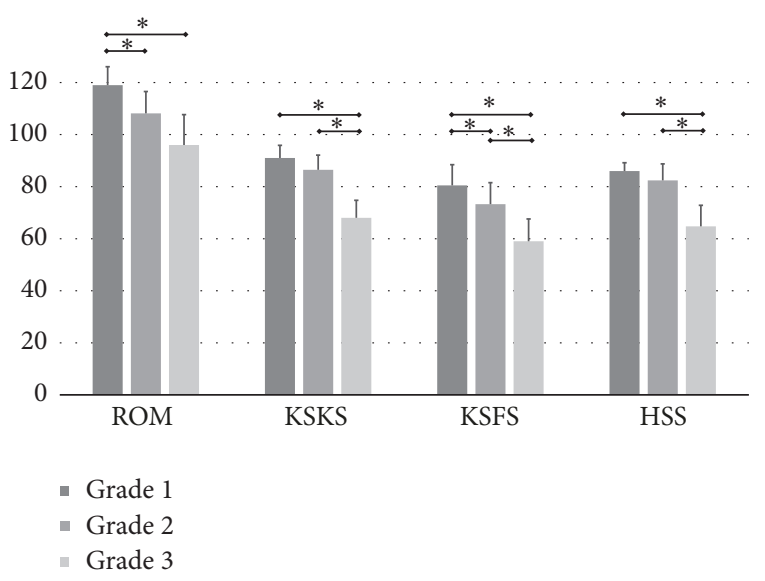

Figure 5: Clinical outcomes according to the severity of the femoral bone defect. Grade 3 bone defects showed inferior outcomes compared to grade 1 and 2 defects. Values are means and standard deviations, and asterisks denote statistical significance. ROM, range of motion; KSKS, Knee Society knee score; HSS, Hospital for Special Surgery knee score.

clinical outcomes in revision TKA. Specifically, the KSKS, KSFS, and HSS scores were related to grade 3 femoral bone defects, ROM and HSS to grade 3 tibial bone defects, and ROM, KSKS, and HSS scores to having three or four surgeries. However, the cause of revision was not associated with the clinical outcomes.

Postrevision clinical outcomes were compared according to bone defect and number of surgeries. As the degree of defective femoral bone increased, the ROM and outcome scores gradually decreased (Figure 5). The clinical outcomes of patients with a grade 3 bone defect were especially poor for every outcome score $(p<0.05)$. Regarding the number of surgeries, three or four surgeries had significantly inferior outcomes $(p<0.05)$. However, patients that had only a twostage revision in the absence of large bone defects did similar to aseptic revisions (Figure 6).

\section{Discussion}

The most important finding of our study was that inferior clinical outcomes in revision TKA surgery were related to large bone defects (grade 3 ) and greater numbers of surgeries (more than three) and not with the type of revision (septic vs. aseptic revision). Septic revision was not directly related to clinical outcomes per se; instead it was indirectly related with an increased number of surgeries and larger bone defects, which are characteristics of persistent infection after failure to control the initial infection. Our data showed that the outcomes of aseptic and septic revision surgery were similar if patients with larger bone defects and more than three surgeries were excluded.

There is debate regarding whether septic revision results in inferior clinical outcomes [2-8, 10-12]. Barrack et al. reported that septic revision was associated with significantly lower functional scores [4]. However, patients undergoing second or third revisions were included. Van Kempen et al.

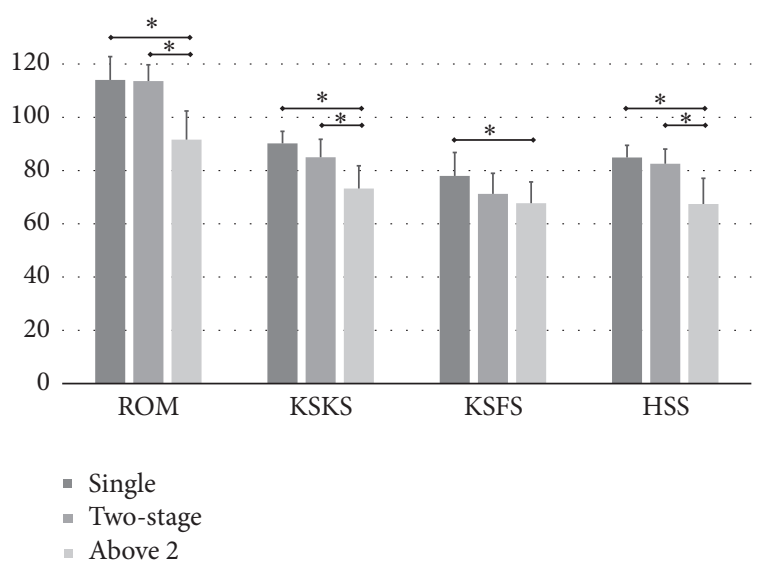

FIGURE 6: Clinical outcomes according to the number of surgeries. Outcomes were inferior with more than two surgeries compared with single- or two-stage surgery. Values are means and standard deviations, and asterisks indicate statistical significance. ROM, range of motion; KSKS, Knee Society knee score; HSS, Hospital for Special Surgery knee score.

reported a similar result, but they did not report on the bone defects encountered or the number of revisions [8]. Patil et al. reported higher clinical scores after septic revisions than after aseptic revisions [11]. However, they included polyethylene exchange and chronic osteomyelitis patients in their analyses. We believe that these inconsistent results originate from the complexity of revision surgery. Most previous reports simply compared septic versus aseptic revision surgery. However, septic revision encompassed various clinical situations, including large bone defects and even failed infection control. It is not "inappropriate" to state that septic revisions have poorer outcomes. That is a fact. However, it is not the infection that is an independent risk factor but rather the size of the bone defect and the number of surgeries.

Limited information is available regarding the factors affecting clinical outcomes in revision surgery. It has been reported that aggressive microorganisms, chronic lymphedema, repeated surgery, and comorbidities increase the failure risk of revision surgery and also result in poor clinical outcomes [18-20]. Although previous studies have focused mainly on the success rate of revision surgery, we believe their findings are in line with our research.

Extra surgical procedures over a two-stage surgery (3rd or 4th surgery) resulted in inferior clinical outcomes. These patients had a decreased ROM as well as poorer functional and pain scores. Repeated tissue injury that results in persistent inflammation with tissue degeneration and emotional depression due to prolonged hospitalization may lead to inferior clinical outcomes for these patients [21-23]. In fact, we often see patients who are depressed and disappointed that the infection was not controlled even after debridement or a two-stage surgery. It is notable that extra surgical procedures (more than two-stage) most commonly occurred due to failed infection control. Sherrell et al. reported that failure of irrigation and debridement leads to subsequent failure of two-stage reimplantation and ultimately requires 
another operation for persistent infection [24]. Our findings suggest that failure to control infection in a two-stage surgery or an inappropriate treatment decision for periprosthetic infection may result in poor clinical outcomes. We would expect satisfactory clinical outcomes for septic revision with a standard treatment protocol.

Several studies have reported poor outcomes for revision TKA of larger bone defects. Franke et al. reported that $20 \%$ of patients experienced a poor outcome in their 5-year followup, and Clatworthy et al. reported a $72 \% 10$-year success rate, meaning that one of four patients required re-revision surgery $[25,26]$. In our case, there were 7 failures among 18 grade 3 femoral bone defects. The most common reason was loosening of the implant, of which there were three cases that eventually required re-revision. The second most common reason was infection. Two patients required arthrodesis. The third most common reason was instability, as two patients required a knee brace but they declined a further procedure. Reconstruction techniques other than an allograft should be considered to solve these problems. Although it is currently unclear why femoral bone defects were more related to a poor outcome than were tibial bone defects, efforts should be made to reduce bone defects, especially of the femur, during revision TKA. In our experience, a motorized burr is better than a curette and osteotome for preserving healthy bone during debridement.

Readers should be aware of several limitations of the current study. First, due to the retrospective nature of the study and the scarcity of revision cases, we could not effectively control the baseline demographics. Thus, gender and BMI in this study differed between the aseptic and septic groups, introducing the possibility of selection bias. Although a matched study would be more desirable, it is actually impossible to match perfectly or stratify subjects while maintaining statistical power in a revision study. Thus, we used multiple regression analysis to correct for confounding bias of independent variables affecting the clinical outcomes. Despite the limitations, the statistical power of our regression model was sufficient to validate our outcome. Second, a considerable amount of variance in our multivariate model remained unexplained. This indicates that other unknown factors, such as combined spine pathology, general health status, quadriceps muscle strength, presence of microorganisms, and mental health, may have been related to the clinical outcomes $[22,23,27,28]$. However, the value of such information is limited as these variables cannot be modified during the surgical procedure. We believe that our evaluation of 13 variables included most of the intraoperative and surgically correctable factors and provided information that was relevant to improving the clinical outcome. Third, the female predominance of the study population should be noted. The proportion of females was $83.8 \%$, which was substantially higher than that reported by other studies of outcomes of revision surgery [2-8, 10-12, 29, 30]. Although there is no clear explanation for the female predominance in knee osteoarthritis, it has been consistently reported in several epidemiologic studies $[31,32]$. This predominance is even greater in Koreans; consequently, the incidence of TKA is 7-8-fold higher in females than in males [33]. This could explain the predominance of females in this study and indicates that the possible selection bias was negligible.

\section{Conclusions}

Clinical outcomes of septic revision were inferior compared to those of aseptic revision. However, poor outcomes mainly resulted from large bone defects and a high number of surgeries. The outcomes of aseptic and septic revision surgery were similar when patients with larger bone defects and more than three surgeries were excluded from the analyses.

\section{Conflicts of Interest}

All authors declare that they do not have any conflicts of interest.

\section{Authors' Contributions}

Du Hyun Ro contributed to planning, statistical analysis, and writing of the manuscript; Jong-Keun Kim contributed to data analysis and revision of the manuscript; Sunghwan Kim contributed to planning and editing of the manuscript; HyukSoo Han contributed to revision of the manuscript and data analysis; Myung Chul Lee contributed to planning, statistical analysis, and writing and editing of the manuscript.

\section{Acknowledgments}

The authors wish to thank Eun Mi Shin and Son Mi Ahn for supporting the research by collecting and organizing the clinical data as well as Eun Soo Ahn for help with proofreading and revising the manuscript.

\section{References}

[1] S. Kurtz, K. Ong, E. Lau, F. Mowat, and M. Halpern, "Projections of primary and revision hip and knee arthroplasty in the United States from 2005 to 2030," The Journal of Bone \& Joint Surgery, vol. 89, no. 4, pp. 780-785, 2007.

[2] K. J. Saleh, D. C. Dykes, R. L. Tweedie et al., "Functional outcome after total knee arthroplasty revision: A meta-analysis," The Journal of Arthroplasty, vol. 17, no. 8, pp. 967-977, 2002.

[3] K. J. Saleh, D. P. Hoeffel, R. A. Kassim, and G. Burstein, "Complications after revision total knee arthroplasty," The Journal of Bone and Joint Surgery-American Volume, vol. 85, pp. 71-74, 2003.

[4] R. L. Barrack, G. Engh, C. Rorabeck, J. Sawhney, and M. Woolfrey, "Patient satisfaction and outcome after septic versus aseptic revision total knee arthroplasty," The Journal of Arthroplasty, vol. 15, no. 8, pp. 990-993, 2000.

[5] C.-J. Wang, M.-C. Hsieh, T.-W. Huang, J.-W. Wang, H.-S. Chen, and C.-Y. Liu, "Clinical outcome and patient satisfaction in aseptic and septic revision total knee arthroplasty," The Knee, vol. 11, no. 1, pp. 45-49, 2004.

[6] S. Y. Pun and M. D. Ries, "Effect of gender and preoperative diagnosis on results of revision total knee arthroplasty," Clinical Orthopaedics and Related Research, vol. 466, no. 11, pp. 27012705, 2008. 
[7] K. Lee, J. Moon, E. Song, H. Lim, and J. Seon, "Minimum twoyear results of revision total knee arthroplasty following infectious or non-infectious causes," Knee Surgery \& Related Research, vol. 24, no. 4, pp. 227-234, 2012.

[8] R. W. T. M. van Kempen, J. J. P. Schimmel, G. G. Van Hellemondt, H. Vandenneucker, and A. B. Wymenga, "Reason for revision TKA predicts clinical outcome: prospective evaluation of 150 consecutive patients with 2-years followup knee," Clinical Orthopaedics and Related Research, vol. 471, no. 7, pp. 22962302, 2013.

[9] D. Johnson and G. Bannister, "The outcome of infected arthroplasty of the knee," The Journal of Bone \& Joint Surgery, vol. 68-B, no. 2, pp. 289-291, 1986.

[10] E. Ghanem, C. Restrepo, A. Joshi, W. Hozack, P. Sharkey, and J. Parvizi, "Periprosthetic infection does not preclude good outcome for revision arthroplasty," Clinical Orthopaedics and Related Research, no. 461, pp. 54-59, 2007.

[11] N. Patil, K. Lee, J. I. Huddleston, A. H. Harris, and S. B. Goodman, "Aseptic versus septic revision total knee arthroplasty: Patient satisfaction, outcome and quality of life improvement," The Knee, vol. 17, no. 3, pp. 200-203, 2010.

[12] A. Rajgopal, A. Vasdev, H. Gupta, and V. Dahiya, "Revision total knee arthroplasty for septic versus aseptic failure.," Journal of Orthopaedic Surgery (Hong Kong), vol. 21, no. 3, pp. 285-289, 2013.

[13] M. D. Wimmer, M. J. Friedrich, T. M. Randau et al., "Polymicrobial infections reduce the cure rate in prosthetic joint infections: outcome analysis with two-stage exchange and follow-up $\geq$ two years," International Orthopaedics, vol. 40, no. 7, pp. 1367-1373, 2016.

[14] M. D. Wimmer, M. M. Ploeger, M. J. Friedrich, T. Hügle, S. Gravius, and T. M. Randau, "Pre-operative intra-articular deep tissue sampling with novel retrograde forceps improves the diagnostics in periprosthetic joint infection," International Orthopaedics, vol. 41, no. 7, pp. 1355-1359, 2017.

[15] V. M. Goldberg, M. P. Figgie, H. E. Figgie III, and M. Sobel, “The results of revision total knee arthroplasty," Clinical Orthopaedics and Related Research, no. 226, pp. 86-92, 1988.

[16] J. K. Lee, S. Lee, D. Kim et al., "Revision total knee arthroplasty with varus-valgus constrained prosthesis versus posterior stabilized prosthesis," Knee Surgery, Sports Traumatology, Arthroscopy, vol. 21, no. 3, pp. 620-628, 2013.

[17] G. A. Engh and D. J. Ammeen, "Bone loss with revision total knee arthroplasty: defect classification and alternatives for reconstruction.," Instructional Course Lectures, vol. 48, pp. 167$175,1999$.

[18] B. Kubista, R. U. Hartzler, C. M. Wood, D. R. Osmon, A. D. Hanssen, and D. G. Lewallen, "Reinfection after two-stage revision for periprosthetic infection of total knee arthroplasty," International Orthopaedics, vol. 36, no. 1, pp. 65-71, 2012.

[19] D. Tigani, G. Trisolino, M. Fosco, R. Ben Ayad, and P. Costigliola, "Two-stage reimplantation for periprosthetic knee infection: influence of host health status and infecting microorganism," The Knee, vol. 20, no. 1, pp. 9-18, 2013.

[20] M. S. Cha, S. H. Cho, D. H. Kim et al., "Two-Stage Total Knee Arthroplasty for Prosthetic Joint Infection," Knee Surgery \& Related Research, vol. 27, no. 2, pp. 82-89, 2015.

[21] J. A. Ulrich, E. C. Liebenberg, D. U. Thuillier, and J. C. Lotz, "ISSLS prize winner: Repeated disc injury causes persistent inflammation," The Spine Journal, vol. 32, no. 25, pp. 2812-2819, 2007.
[22] J. A. Singh and D. Lewallen, "Age, gender, obesity, and depression are associated with patient-related pain and function outcome after revision total hip arthroplasty," Clinical Rheumatology, vol. 28, no. 12, pp. 1419-1430, 2009.

[23] J. A. Singh and D. G. Lewallen, "Medical and psychological comorbidity predicts poor pain outcomes after total knee arthroplasty," Rheumatology, vol. 52, no. 5, pp. 916-923, 2013.

[24] J. C. Sherrell, T. K. Fehring, and S. Odum, "The chitranjan ranawat award: fate of two-stage reimplantation after failed irrigation and débridement for periprosthetic knee infection," Clinical Orthopaedics and Related Research, vol. 469, no. 1, pp. 18-25, 2011.

[25] M. G. Clatworthy, J. Ballance, G. W. Brick, H. P. Chandler, and A. E. Gross, "The use of structural allograft for uncontained defects in revision total knee arthroplasty: A minimum fiveyear review," The Journal of Bone \& Joint Surgery, vol. 83, no. 3, pp. 404-411, 2001.

[26] K. F. Franke, I. Nusem, G. Gamboa, and D. A. F. Morgan, "Outcome of revision total knee arthroplasty with bone allograft in 30 cases," Acta Orthopeedica Belgica, vol. 79, no. 4, pp. 427-434, 2013.

[27] D. J. Laudermilch, C. J. Fedorka, A. Heyl, N. Rao, and R. L. McGough, "Outcomes of revision total knee arthroplasty after methicillin-resistant staphylococcus aureus infection," Clinical Orthopaedics and Related Research, vol. 468, no. 8, pp. 20672073, 2010.

[28] J. A. Singh, M. M. O’Byrne, W. S. Harmsen, and D. G. Lewallen, "Predictors of moderate-severe functional limitation 2 and 5 years after revision total knee arthroplasty," The Journal of Arthroplasty, vol. 25, no. 7, pp. 1091-1095.e4, 2010.

[29] R. T. Goldman, G. R. Scuderi, and J. N. Insall, "2-Stage reimplantation for infected total knee replacement," Clinical Orthopaedics and Related Research, no. 331, pp. 118-124, 1996.

[30] A. A. Haleem, D. J. Berry, and A. D. Hanssen, "Mid-term to long-term followup of two-stage reimplantation for infected total knee arthroplasty," Clinical Orthopaedics and Related Research, no. 428, pp. 35-39, 2004.

[31] Centers for Disease Control and Prevention, "Racial disparities in total knee replacement among Medicare enrollees-United States, 2000-2006," Morbidity and Mortality Weekly Report, vol. 58, no. 6, pp. 133-138, 2009.

[32] Y. Zhang and J. M. Jordan, "Epidemiology of osteoarthritis," Clinics in Geriatric Medicine, vol. 26, no. 3, pp. 355-369, 2010.

[33] H.-A. Kim, S. Kim, Y. I. Seo et al., "The epidemiology of total knee replacement in South Korea: national registry data," Rheumatology, vol. 47, no. 1, pp. 88-91, 2008. 


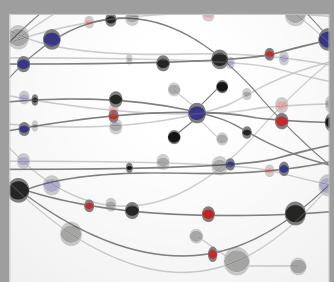

The Scientific World Journal
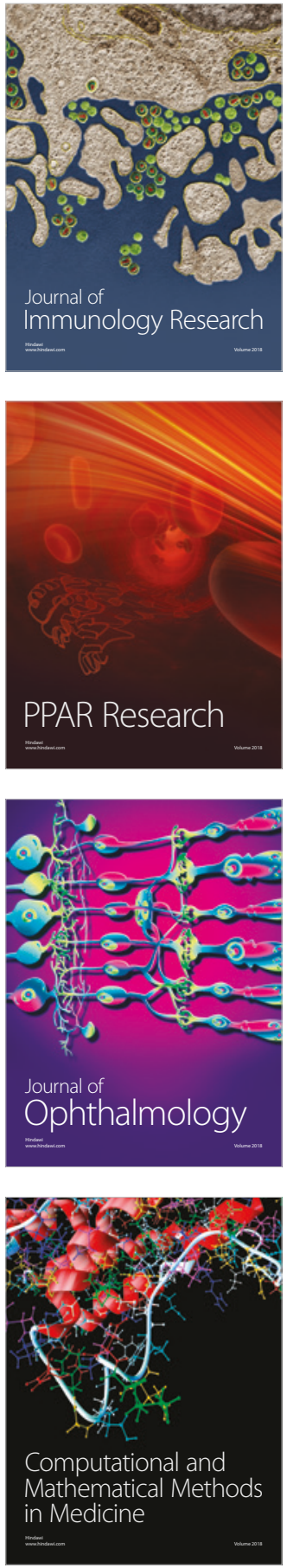

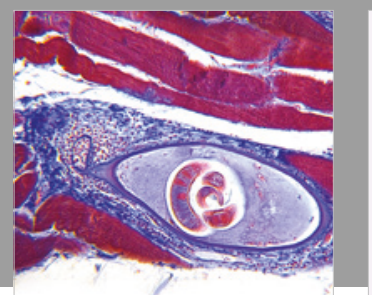

Gastroenterology Research and Practice

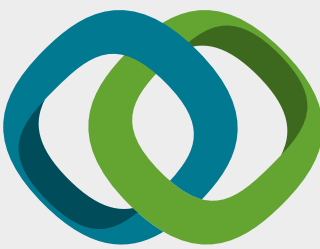

\section{Hindawi}

Submit your manuscripts at

www.hindawi.com
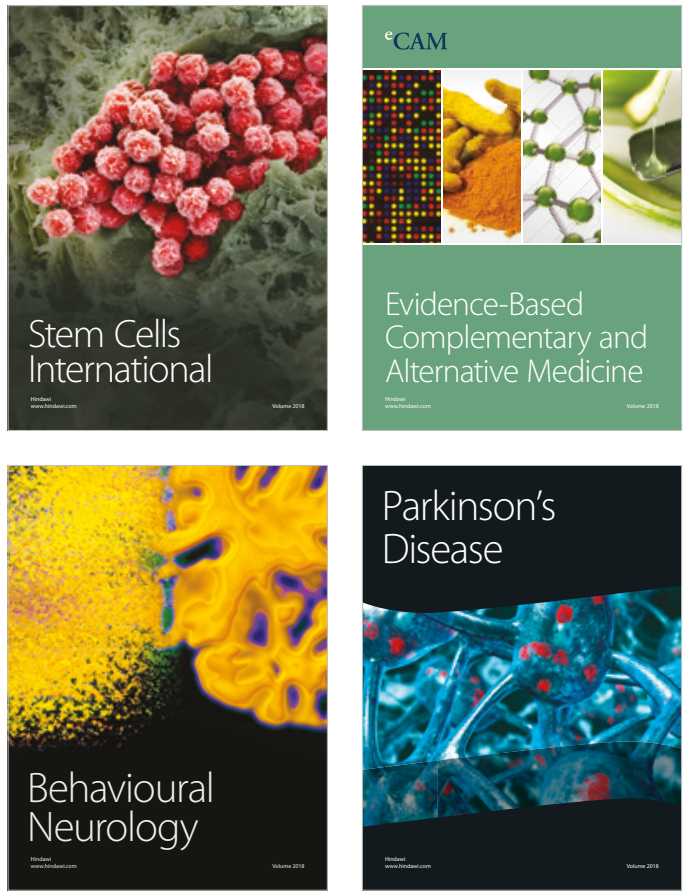

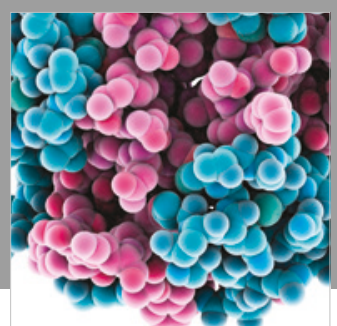

ournal of

Diabetes Research

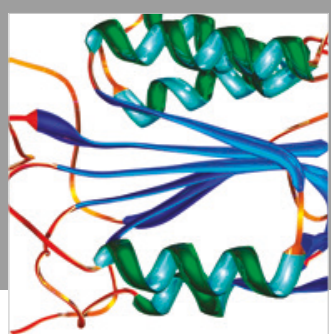

Disease Markers
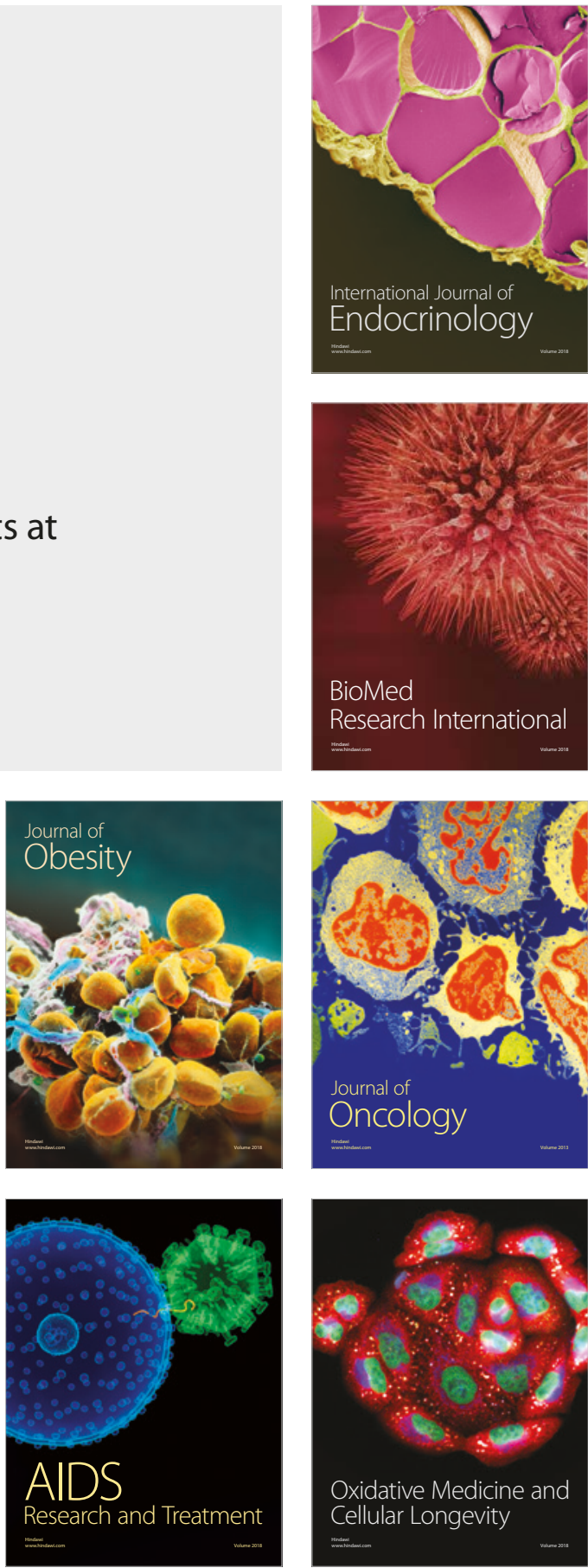